\title{
CARACTERIZACIÓN DE LOS HÁBITOS DE DOCUMENTACIÓN TERMINOLÓGICA DE LOS ESTUDIANTES DE TRADUCCIÓN
}

\author{
Alicia Bolaños-Medina \\ Universidad de Las Palmas de Gran Canaria \\ abolanos@dfm.ulpgc.es \\ Ana-María Monterde-Rey \\ Universidad de Las Palmas de Gran Canaria \\ amonterde@dfm.ulpgc.es
}

\begin{abstract}
Resumen: En este artículo presentamos una investigación preliminar realizada con estudiantes de Traducción e Interpretación para determinar sus hábitos de documentación terminológica. La experiencia consistió en un ejercicio en el que se les pedía que consultaran las fuentes documentales de Internet que consideraran oportunas para encontrar la equivalencia al español de términos ingleses sobre cardiología; y en rellenar un cuestionario sobre diversos aspectos relacionados con su perfil, sus hábitos documentales y la tarea en sí. Para recabar la información sobre los pasos seguidos por los participantes a la hora de documentarse en Internet, se utilizó un software de seguimiento de la actividad realizada en cada uno de los equipos. El análisis de los datos así recogidos nos permite trazar el perfil de documentación terminológica de los estudiantes. Un objetivo secundario ha sido evaluar la utilidad y metodología de uso de la herramienta informática de seguimiento de la actividad utilizada.
\end{abstract}

Palabras clave: Documentación terminológica, documentación, terminología, traducción. 


\title{
CHARACTERIZATION OF TRANSLATION'S STUDENTS HABITS OF TERMINOLOGICAL DOCUMENTATION
}

\begin{abstract}
In this paper we present preliminary research with students of Translation and Interpreting to determine their habits of terminological documentation. This experience consisted of two exercises: 1) to look for the internet sources that students thought to be more adequate to find the Spanish terminological equivalent of English terms on Cardiology, and 2) to fill up a questionnaire on different aspects of their profile, their habits on documentation and the task itself. Keylogger software was used to know the steps that students followed to look for documents on the Internet. Analysis of these data will allow us to determine the students' terminological documentation profile and to evaluate the utility and methodology of use of the keylogger software
\end{abstract}

Keywords: Terminological documentation, documentation, terminology, translation.

\section{Introducción}

La terminología es esencial en la traducción especializada. De hecho, es uno de los elementos característicos que diferencian los textos generales de los especializados (Arntz y Picht, 1995, p. 43). Picht (1998, inédito) afirma que hasta un $85 \%$ del vocabulario de un documento especializado puede ser terminología y el $50 \%$ del tiempo invertido en una traducción científico-técnica se emplea en la investigación terminológica (Picht, 1992, p. 38). Una buena traducción debe ser precisa desde el punto de vista terminológico (Cabré, 1999, p. 204), por lo que dos de los principales obstáculos en la traducción científico-técnica son la búsqueda de términos equivalentes en la lengua meta y la comprensión de los conceptos que representan. Esta búsqueda puede ser problemática por varios motivos: por existir vacíos conceptuales o terminológicos en la lengua de llegada; por el desconocimiento del área en la que va a traducir, etc. Todos estos problemas, sin embargo, se pueden solucionar gracias a la documentación. 
La documentación es básica para la terminología, pues aporta los materiales necesarios para la realización de trabajos terminológicos y para la adquisición de conocimientos (Cabré y Codina, 2001, p.15). La terminología necesita documentos terciarios en los que encontramos terminología y relaciones conceptuales; documentos primarios que, además de contener terminología, permiten adquirir competencia cognitiva sobre una materia o verificar informaciones; y documentos secundarios que nos permiten acceder a los documentos primarios y terciarios.

Por último, la imbricación entre documentación y terminología es indiscutible. Ambas disciplinas se relacionan de forma bidireccional y así lo han resaltado numerosos autores (Wüster, 1969; Arntz y Picht, 1995; Irazazábal Nerpell, 1996; Cabré, 1993, 1999, entre otros). A través del trabajo terminográfico sistemático, la terminología produce documentos que pasan a formar parte de la documentación. Por tanto, podemos concluir diciendo que no se puede hacer terminología sin la ayuda de la documentación, pues es uno de los pilares del trabajo terminológico.

En los últimos años, Internet se ha convertido en la herramienta de consulta terminológica y documental por excelencia del traductor, pero también presenta una enorme "dispersión de datos poco estructurados" (Pinto, 2000, p. 6), lo que puede incidir negativamente en los resultados del proceso documental y conlleva a menudo una gran pérdida de tiempo. Asimismo, es necesario usar las fuentes de Internet con precaución, pues "busque lo que busque el traductor lo encuentra, tanto si busca una expresión correcta como incorrecta" (Sevilla Muñoz y Sevilla Muñoz, 2005). Según Cid Leal y Recoder Sellarés (2000, p. 53), el éxito de la búsqueda documental de un traductor en Internet dependerá de: la correcta identificación del problema de traducción, el grado de accesibilidad de las diferentes fuentes de información, el tiempo disponible, sus conocimientos previos sobre el tema, y, por último, pero no menos importante, de su habilidad para recuperar la información de la red.

Teniendo en cuenta este último punto, hemos llevado a cabo un experimento preliminar durante una sesión de la asignatura de 
terminología de la Facultad de Traducción e Interpretación (FTI) de la Universidad de Las Palmas de Gran Canaria (ULPGC). El propósito fundamental de este estudio descriptivo es obtener información sobre los hábitos de búsqueda documental terminológica de los estudiantes de último curso de licenciatura de Traducción e Interpretación. Un objetivo secundario ha sido evaluar la utilidad y la metodología de uso de la herramienta informática de seguimiento de la actividad utilizada.

Los resultados obtenidos sobre el perfil de uso de las fuentes documentales de Internet por parte de los estudiantes pueden contribuir a identificar sus carencias e, indirectamente, a mejorar el diseño de los proyectos docentes de la materia de documentación en las facultades de traducción. Se trata de algo fundamental si atendemos a que se sigue constatando tanto la necesidad de diseñar cursos de documentación para traductores aún más específicos (Mayoral Asensio, 1997-1998) como la carencia de estrategias didácticas que desarrollen mejor su competencia en este ámbito (SánchezValverde, 2007).

\section{Investigaciones previas sobre el proceso de document- ación en la búsqueda terminológica y documental de los estudiantes de traducción.}

La función de la documentación es tan determinante que "la disponibilidad de fuentes de información puede condicionar la forma de traducir" (Mayoral Asensio, 1997-1998, p. 14). Sin embargo, a pesar de que el proceso de documentación ha sido objeto de algunas investigaciones, "persiste la escasez de recursos y métodos didácticos que verdaderamente ayuden al traductor a mejorar sus destrezas en documentación" (Sánchez Valverde, 2007, p. 11). Siendo así, parece lógico que se deba prestar especial atención a la investigación del proceso de documentación en sí y a las posibles estrategias documentales válidas. 
El estudio del uso de los diccionarios por parte del traductor ha generado el mayor número de trabajos en este campo. Un metaanálisis realizado por Rodrigues (2008), concluye, tras revisar varias investigaciones (Krings, 1986; Königs, 1989; Hönig, 1988; Gerloff, 1988; Jääskeläinen, 1989; Kußmaul, 1995; Kiraly, 1995; entre otros) que, mientras que los estudiantes en lenguas extranjeras utilizan poco los diccionarios, quizá por interpretar su uso como señal que pone de manifiesto sus carencias lingüísticas, los traductores profesionales y los estudiantes de traducción se sirven de ellos en mayor medida. Los estudios revisados por Rodrigues también muestran la existencia de ciertas estrategias de uso de cara a la traducción, como la verificación de un término dado en varios diccionarios o en un diccionario monolingüe para encontrar un sinónimo.

Kunzli (2001) realizó un estudio con seis sujetos, tres traductores profesionales y tres estudiantes de traducción, mediante protocolo de verbalización y entrevista, que permitía la consulta de información en soporte digital. Encontró que existía una correlación positiva entre el número de fuentes de información consultadas, la experiencia y la calidad de la traducción.

En la misma línea de investigación aplicada a la didáctica de la traducción, Kim (2006) efectuó una investigación con 32 sujetos, estudiantes de traducción entre las lenguas coreana e inglesa. Analizó si el hecho de tener acceso previamente a información de apoyo sobre el tema de la traducción influía o no en los resultados, y si su calidad y su cantidad eran determinantes o no. Los resultados sugieren que el hecho de disponer de información de apoyo influye en mayor medida incluso que el nivel de destreza lectora en inglés; también se comprobó que mientras que la calidad de la información de apoyo es un factor determinante, no lo es su cantidad.

Sánchez Valverde (2007) atribuye la escasez de investigaciones empíricas en este ámbito a las limitaciones metodológicas de recogida de datos, ya que tradicionalmente se han venido utilizando únicamente protocolos verbales y entrevistas. Sin embargo, en los últimos años, al tiempo que Internet y el formato digital se consolidaban como los soportes documentales más utilizados, han apa- 
recido nuevas herramientas que permiten ampliar los enfoques de investigación. La propia Sánchez-Valverde presentó el diseño de un estudio para investigar las estrategias de búsqueda de información de los sujetos en Internet, desde un enfoque cognitivo (op. cit.). El objetivo último de este proyecto era la creación de una herramienta pedagógica y recogía el uso de programas de captura de las acciones realizadas por cada participante en un equipo informático, tales como Translog 2000, creado específicamente para la investigación empírica en traducción; Camtasia, para grabar en vídeo la pantalla del ordenador; y KGB Spy, de funcionamiento similar al utilizado en la experiencia que describimos en este artículo (veáse epígrafe 3.2).

\section{Metodología}

\subsection{Condiciones experimentales e instrumentos de recogida de datos}

El experimento, consistió en la cumplimentación de un ejercicio supervisado mediante un formulario, en el que se pedía a los estudiantes que consultaran las fuentes documentales de Internet que consideraran oportunas para encontrar la equivalencia al español de nueve términos médicos especializados en inglés, relacionados con la cardiología. La duración de la prueba, realizada en una sesión de clase, fue de una hora. Los estudiantes accedieron a ella mediante conexión al espacio reservado a la asignatura en el campus virtual mediante la plataforma Moodle y accedieron a Internet. Tras la realización de la tarea, rellenaron un cuestionario idéntico con tres bloques de preguntas relacionados con su perfil, sus hábitos documentales cotidianos y la tarea. El número de ítems que debían rellenar se limitó a 18 para evitar los sesgos motivacionales y atencionales propios de cuestionarios más largos, sobre todo después de haber realizado una actividad que exige concentración intensa. 
Para recabar la información sobre los pasos seguidos por los participantes para documentarse en Internet, se utilizó el programa de Refog ${ }^{1}$, Key Logger 5.1. (véase apartado 4.7). En el momento de la recogida de la información, los participantes desconocían que se iba a registrar cada una de sus acciones; esto fue así para evitar posibles sesgos en los resultados. Tras la finalización del experimento, todos fueron informados de sus objetivos y metodología y se les explicó que el procesamiento de la información se realizaría de forma anónima y serviría únicamente para los fines de la investigación; en ningún caso influiría en la evaluación académica de la asignatura. Por último, se les dio la oportunidad de renunciar formalmente a participar en el estudio, pero ningún estudiante optó por esta última vía.

\subsection{Análisis de la muestra}

Realizaron la prueba un total de 58 estudiantes matriculados en la asignatura de terminología, de cuarto año de titulación en Traducción e Interpretación, en el curso 2009-2010. Sin embargo, 17 de ellos no pudieron ser tenidos en cuenta por diversos motivos: la mayoría, por no haber cumplimentado todos los documentos necesarios; por provenir de universidades extranjeras; por no ser su lengua materna el castellano; o por motivos técnicos.

Así, la muestra final está formada por 40 estudiantes. La media de edad es de 23,3 años, con una moda de 21 años y una desviación típica de 4,26 . De todos ellos, nueve eran varones, frente a 31 mujeres. Esta ratio de marcada mayoría femenina es frecuente en los estudios de esta rama. Un total de 38 estudiantes tenían el inglés como primer idioma de estudio; los otros dos estudiaban el alemán como lengua principal, pero también cursaban inglés como segundo idioma.

\subsection{Análisis de datos}

Tras realizar un primer análisis de los datos recopilados con los instrumentos de recogida de información de un $10 \%$ de la muestra, 
con el fin de evaluar las vías de valoración más apropiadas, dadas las características de los datos, y refinar así la metodología inicialmente planteada, los formularios fueron vaciados en una hoja de cálculo. Se utilizaron las aplicaciones Excel de Microsoft y PASW Statistics 17.0, de SPSS. Se han realizado análisis estadísticos descriptivos de todas las variables y se han hallado las correlaciones de Pearson entre las más representativas.

\section{Resultados}

\subsection{Caracterización general}

El número medio de horas de uso de Internet a la semana, sin contar las dedicadas a utilizar herramientas de chat, fue de 10,8; en este caso, a pesar de que ha existido una gran variabilidad (con desviación típica de 7,9), la moda fue de 10 horas, aunque el número mínimo de horas reseñado osciló entre 0,5 y 35 . Nos ha parecido relevante tener en cuenta estos datos porque implican una variabilidad individual considerable que no debe ser desestimada.

En cuanto a la valoración realizada por los sujetos de sus conocimientos previos sobre el tema del que trataba la traducción, tanto la media como la moda se situaron en cuatro puntos, pero con una varianza considerable (la desviación típica fue de 2,12); el valor mínimo reseñado fue cero y el máximo 7,5.

\subsection{Hábitos generales de consulta de documentación im- presa y digital}

Los sujetos declararon utilizar de forma habitual hasta casi cuatro veces más las fuentes de documentación de Internet que las tradicionales impresas. Llaman la atención dos hechos: en primer lugar, que el porcentaje de uso de las fuentes en papel en ningún caso sobrepasó el $50 \%$; en segundo lugar, que, en contrapartida, el 
porcentaje de utilización de fuentes digitales declarado nunca fue inferior al 50\%. En la tabla 1 se detalla más información.

\begin{tabular}{|l|l|l|}
\hline & Impresa & Digital \\
\hline Media & 19,29 & 81,90 \\
\hline Moda & 10,00 & 80,00 \\
\hline Desviación típica & 12,65 & 13,26 \\
\hline Rango & 49,99 & 50,00 \\
\hline Mínimo & 0,01 & 50,00 \\
\hline Máximo & 50,00 & 100,00 \\
\hline
\end{tabular}

Tabla 1. Porcentaje de uso de documentación en papel y digital

En cuanto a las fuentes de documentación de Internet o en soporte digital más usadas, todos los participantes afirmaron utilizar glosarios, diccionarios y textos paralelos digitales, el 52\% consulta también bases y bancos de datos y el $45 \%$, se sirve de los grupos de noticias. Las fuentes de Internet con menor frecuencia de uso son las memorias de traducción $(7,5 \%)$ y las listas de correo $(2,5)$.

\subsection{Autoeficacia percibida}

La autoeficacia elevada se ha asociado con la realización de un esfuerzo de mayor envergadura y la mayor persistencia en la consecución de una meta así como un afrontamiento más eficaz a la hora de realizar tareas en condiciones que pueden resultar estresantes (Goddard et al., 2004; Huici y Morales, 2004).

En cuanto a la autoeficacia percibida sobre el uso del ordenador, en una escala del uno al diez, la media fue de 7,7. Asimismo, las medias informadas sobre autoeficacia a la hora de documentarse en Internet para traducir, en general, y sobre su autoeficacia específica percibida durante la realización de la tarea propuesta se situaron también entre siete y ocho puntos, aunque se observa una mayor variabilidad de las puntuaciones en la evaluación de la 
destreza de uso del ordenador. En la tabla 2 se ofrece información más detallada sobre estos tres parámetros.

\begin{tabular}{|l|l|l|l|}
\hline & Ordenador & Internet & Prueba \\
\hline Media & 7,68 & 7,32 & 7,59 \\
\hline Moda & 7,00 & 7,00 & 8,00 \\
\hline Desviación típica & 1,43 & 0,88 & 1,16 \\
\hline Rango & 6,00 & 3,00 & 5,00 \\
\hline Mínimo & 4,00 & 6,00 & 5,00 \\
\hline Máximo & 10,00 & 9,00 & 10,00 \\
\hline
\end{tabular}

Tabla 2. Autoeficacia percibida al usar el ordenador, documentarse en Internet y realizar esta prueba.

\subsection{Fuentes consultadas}

Cada estudiante consultó una media de 11,10 fuentes documentales de Internet diferentes para encontrar soluciones de traducción adecuadas para los términos propuestos, siendo el máximo alcanzado 20 fuentes diferentes por sujeto (véase tabla 3 ).

\begin{tabular}{|l|l|}
\hline & $\mathrm{N}^{\mathrm{o}}$ de fuentes \\
\hline Media & 11,11 \\
\hline Moda & 13,00 \\
\hline Desviación típica & 3,94 \\
\hline Rango & 15,00 \\
\hline Mínimo & 5,00 \\
\hline Máximo & 20,00 \\
\hline
\end{tabular}

Tabla 3. Fuentes consultadas

En total, los sujetos consultaron 132 fuentes documentales distintas. En la tabla 4, se recogen las 22 fuentes más utilizadas (que 
acumulan el $84,23 \%$ de las consultas), es decir, todas aquellas que han superado un $0,5 \%$ de utilización. Llama la atención que cuatro fuentes documentales han acaparado casi el 52\% de las consultas y que sólo una de ellas es de carácter especializado: el diccionario Wordreference, la pagina web de Wikipedia, tanto en inglés como en español (aunque se ha utilizado en mayor medida la versión en lengua inglesa) y MedlinePlus, la página web de información médica de la Biblioteca Nacional de Medicina y los Institutos Nacionales de la Salud de Estados Unidos. De todas las fuentes recogidas en la tabla 4, sólo la mitad (11 de ellas) contienen específicamente información sobre medicina (véase tabla 4). Asimismo, cabe destacar que 59 de las fuentes recopiladas fueron consultadas en una única ocasión.

\begin{tabular}{|l|l|}
\hline Fuentes bibliográficas & $\%$ \\
\hline Wordreference.com & 15,77 \\
\hline Wikipedia.en & 14,21 \\
\hline Medline plus & 10,90 \\
\hline Wikipedia.es & 10,90 \\
\hline Freedictionary.com Medical Dictionary & 6,49 \\
\hline Reverso.net [collins lexibase] & 5,71 \\
\hline Proz.com & 5,39 \\
\hline Medterms.com & 2,21 \\
\hline RAE & 1,36 \\
\hline Diagnosticomedico.es & 1,30 \\
\hline es.diagnosispro.com & 1,17 \\
\hline Babylon.com (bilingüe) & 1,04 \\
\hline Wordmagicsoft & 1,04 \\
\hline 123teachme medical dictionary & 0,97 \\
\hline Merriam-webster.com & 0,97 \\
\hline IQB.es (diccionario) & 0,91 \\
\hline Medilexicon.com & 0,78 \\
\hline en.diagnosispro.com & 0,71 \\
\hline
\end{tabular}




\begin{tabular}{|l|l|}
\hline IATE & 0,71 \\
\hline Medciclopedia.com & 0,65 \\
\hline Dicciomed.es & 0,52 \\
\hline Medicaldoctordictionary.com & 0,52 \\
\hline
\end{tabular}

Tabla 4. Fuentes bibliográficas de Internet más consultadas por los estudiantes.

Con el objeto de comparar estos resultados con los que presentamos en el siguiente epígrafe, resumimos la tabla anterior con las fuentes más consultadas agrupadas por temática general. A pesar de ser Medline Plus una web especializada de medicina, la hemos considerado en un apartado distinto, por el gran número de consultas recibidas.

\begin{tabular}{|l|l|}
\hline Fuentes bibliográficas & $\%$ \\
\hline Diccionarios & 26,8 \\
\hline Wikipedia & 25,11 \\
\hline MedlinePlus & 10,90 \\
\hline Diccionarios médicos & 9,28 \\
\hline Webs especializadas medicina & 5,39 \\
\hline Proz.com & 5,39 \\
\hline Enciclopedias especializadas & 0,65 \\
\hline IATE & 0,71 \\
\hline
\end{tabular}

Tabla 5. Fuentes bibliográficas de Internet más consultadas por los estudiantes.

\subsection{Fuentes consideradas más fiables}

Tras realizar la prueba, también se interrogó a los alumnos sobre cuál era para ellos la fuente más fiable que habían consultado. El resultado fue el mostrado en la siguiente tabla: 


\begin{tabular}{|l|l|}
\hline Fuentes bibliográficas & $\%$ \\
\hline Webs especializadas en medicina & 25 \\
\hline Diccionarios médicos & 20 \\
\hline MedlinePlus & 12,5 \\
\hline Wikipedia & 7,5 \\
\hline Vademécum & 5 \\
\hline Proz.com & 5 \\
\hline Diccionarios & 5 \\
\hline Otras $^{2}$ & 17,5 \\
\hline
\end{tabular}

Tabla 6. Fuentes bibliográficas de Internet valoradas más fiables

Como se puede comprobar, las fuentes bibliográficas valoradas más fiables han sido las webs especializadas en medicina, seguidas de los diccionarios médicos. Si lo comparamos con los resultados del epígrafe anterior, podemos concluir que, exceptuando el caso de MedlinePlus, no se corresponden las fuentes más consultadas con las más fiables.

\subsection{Rendimiento y relaciones entre variables}

Se ha valorado el grado de adecuación de las soluciones de traducción al castellano propuestas por los sujetos para cada uno de los nueve términos usados como reactivos, mediante la asignación de una puntuación acorde, y se ha hallado una calificación media del rendimiento de cada sujeto que se sitúa en una escala del 1 al 9. Sin embargo, hemos encontrado que estos datos no arrojan demasiada luz, ya que, a pesar de que la mayoría de los participantes declara tener conocimientos previos sobre el tema de la traducción inferiores a 5 puntos, la calificación media obtenida es de 7,97. El hecho de haber traducido textos médicos especializados con anterioridad no se refleja prácticamente en el rendimiento obtenido en la prueba, pero sí en cuanto al número de fuentes consultadas se refiere; en 
el caso de los estudiantes que ya contaban con experiencia en este ámbito, la media de direcciones URL consultadas asciende a 9,9, frente a las 12,12 de los que no disponían de dicha experiencia.

No se ha constatado relación entre la calificación obtenida en la prueba y el porcentaje de asignaturas de la licenciatura aprobadas previamente $(\mathrm{r}=0,10)$, ni tampoco entre éste último y el número de fuentes diferentes consultadas por los sujetos $(r=0,014)$. El rendimiento de los sujetos que ya habían aprobado otra asignatura de traducción científica y técnica no difiere significativamente del de los que aún no lo habían hecho. Por último, el número de horas por semana dedicadas a navegar por Internet tampoco parece guardar relación directa con ninguno de los factores analizados.

\begin{tabular}{|l|l|l|l|l|l|}
\hline & $\begin{array}{l}\text { Efic. } \\
\text { Internet }\end{array}$ & $\begin{array}{l}\text { Efic. } \\
\text { prue- } \\
\text { ba }\end{array}$ & $\begin{array}{l}\text { Efic. } \\
\text { ordena- } \\
\text { dor }\end{array}$ & $\begin{array}{l}\text { C a - } \\
\text { lif } \\
\text { F i - } \\
\text { nal }\end{array}$ & $\begin{array}{l}\text { Fuentes } \\
\text { diferentes }\end{array}$ \\
\hline Efic. Internet & 1 & & & & \\
\hline Efic. prueba & 0,52 & 1 & & & \\
\hline Efic. ordenador & 0,62 & 0,50 & 1 & & \\
\hline Rendimiento & 0,50 & 0,37 & 0,42 & 1 & \\
\hline $\begin{array}{l}\text { Fuentes diferen- } \\
\text { tes }\end{array}$ & 0,10 & 0,01 & 0 & 0,05 & 1 \\
\hline
\end{tabular}

Tabla 7. Coeficientes de correlación hallados entre diferentes variables.

Como mostramos en la tabla 7 , se ha obtenido una correlación significativa entre los grados de autoeficacia informada sobre el uso del ordenador y sobre la capacidad de documentarse en Internet para realizar una traducción $(\mathrm{r}=0,62)$. No se han observado correlaciones lineales positivas entre el número de fuentes utilizadas y el rendimiento. 


\subsection{Evaluación del uso de la herramienta informática de seguimiento de la actividad}

A continuación, recogemos las principales conclusiones obtenidas del uso de las diferentes características del programa Refog Keylogger.

El archivo recopilatorio de capturas de pantalla del ordenador de cada participante fue preferible a la captura restringida a la ventana activa, ya que permitió acceder a otro tipo de información (por ej., cuántas aplicaciones se estaban utilizado a la vez), como también lo fue realizar las capturas de pantalla cada vez que se abría una ventana nueva, frente a la opción de hacerlas cuando el usuario hiciera clic con el ratón. El ajuste de la calidad de las capturas de pantalla en un grado medio fue útil para controlar el volumen que ocupa la información en el disco.

Los datos recogidos en los informes de los sitios web visitados por cada sujeto (fecha y hora de consulta, título de la ventana, URL visitada, navegador usado y captura de pantalla) durante la hora de ejecución de la prueba resultaron suficientes. Dependiendo del fin de la investigación, se puede preferir una de las dos opciones del programa, ya sea el registro de toda la navegación realizada en un sitio web dado (si se pretende reconstruir paso a paso la estrategia seguida intrasujeto, por ejemplo, para su posterior comparación intersujetos) o sólo de la página principal de éste (si lo fundamental es determinar la variedad de URL consultadas). En esta fase preliminar del estudio utilizamos éste último.

En todo momento resultó necesario encontrar un punto de equilibrio entre la "informatividad" de los datos recopilados y la "sobreinformación", por ejemplo, para establecer los intervalos de tiempo entre una captura de pantalla y la siguiente, que pueden oscilar desde un segundo hasta varios minutos. Un intervalo muy corto puede entorpecer la tarea de distinguir qué registros son significativos para el estudio, y uno muy largo puede pasar por alto acciones relevantes del usuario. 
Por último, el registro de todas las pulsaciones de teclas recogió la fecha y hora, la aplicación informática, el título de la ventana, el número de teclas pulsadas y el texto exacto introducido, resultó especialmente útil para diferenciar entre las fuentes de Internet que ya conocían los usuarios y aquéllas que encontraban al utilizar buscadores generales.

Una gran ventaja de esta herramienta es que se ejecuta en modo oculto y no es posible detectarlo ni en el escritorio, ni en el área de notificación, ni en el administrador de tareas, ni en el menú de inicio de Windows; ningún sujeto sospechó que sus acciones estaban siendo grabadas.

\section{Conclusiones}

Los estudiantes de traducción-interpretación declaran usar hasta casi cuatro veces más las fuentes de documentación en Internet que las impresas en su quehacer diario, sobre todo glosarios, diccionarios y textos paralelos; las menos usadas, con gran diferencia, son las memorias de traducción y las listas de correo, sobre las que cabría hacer hincapié en el marco del proyecto docente de la asignatura. Presentan una gran dispersión en la búsqueda documental. Además, a pesar de buscar términos, apenas consultan fuentes especializadas y algunas a las que acceden son de dudosa calidad, lo que prueba, por ejemplo, el uso generalizado de Wikipedia, tanto en español como en inglés, como uno de los cuatro recursos más empleados. Este hecho se debe, quizá, a la gran popularidad que esta herramienta tiene entre la población juvenil y que, al tratarse de una enciclopedia, en principio, podría recoger cualquier palabra o término. Las fuentes más consultadas no se corresponden con las fuentes que consideran más fiables. Existe correlación entre la autoeficacia percibida a la hora de utilizar el ordenador y la que se atribuyen los sujetos para realizar el proceso de documentación previo a la traducción. El tener conocimientos previos sobre el 
campo del saber al que pertenecen los términos de búsqueda no determina el rendimiento, pero sí el número de fuentes consultadas, que es menor. Quizá esto puede deberse también al hecho de conocer de antemano la existencia de ciertas fuentes contrastadas especializadas en el área temática en cuestión, por lo que se dirigen directamente a ellas en vez de realizar búsquedas previas para intentar localizarlas. Esto no concuerda con los datos hallados por Kunzli (2001) que encontró que sí existe una correlación positiva entre el número de fuentes de información consultadas, la experiencia y la calidad de la traducción. Se trata de un aspecto que, sin duda, requiere de más investigación. El hecho de no haber hallado correlaciones significativas entre el rendimiento relativamente elevado en la prueba y otros factores como la experiencia previa en traducción científica o el número de asignaturas de la carrera ya aprobadas, entre otros, podría ser indicativo de la solidez de la competencia documental general adquirida por los estudiantes durante los 3 años anteriores de carrera o relacionarse con el grado medio de dificultad de la prueba. Una vez más, se hace necesario investigar más al respecto.

En cuanto a la metodología empleada para la recogida de datos, podemos decir que, en general, el grado de detalle de los documentos generados automáticamente por la aplicación Refog Key Logger ha resultado adecuado para los fines de la investigación y se valora positivamente la transparencia de su uso para los participantes en el estudio, que en ningún momento sospecharon que sus movimientos estaban siendo grabados, lo que repercute positivamente en la validez ecológica del estudio. Sin embargo, disminuir el intervalo de tiempo entre las capturas de pantalla hasta lograr una mejor ratio entre la "informatividad" y la "sobreinformación" o utilizar un programa de grabación en vídeo de la pantalla del ordenador, podrían facilitar aún más la reconstrucción del proceso y disminuir el tiempo empleado en el análisis, por lo que es necesario mayor investigación. 
A pesar de sus limitaciones, dadas en su mayoría por su carácter preliminar, este trabajo ha contribuido, en cierta medida, a colmar la laguna bibliográfica detectada en cuanto a estudios descriptivos del proceso de documentación terminológica en Internet y ha permitido evaluar el uso de una nueva metodología de recogida de datos prometedora de cara a futuras investigaciones. Asimismo, pretendemos seguir explorando en el futuro, con muestras más amplias, otros aspectos, tales como si las tendencias detectadas se mantienen en otros campos especializados y en un periodo de tiempo más o menos prolongado, ante la rápida evolución de los formatos documentales de Internet.

Recebido em 25/03/11

Aceito em 21/11/11

Notas

1. Se puede encontrar más información en http://www.refog.es/.

2. Las siguientes fuentes solo fueron seleccionadas por un alumno: textos paralelos, contextos médicos con resúmenes, glosario del manual Merk, revistas médicas, IATE, diccionario Babylon y Word Magic, enciclopedias. 


\section{Bibliografia}

ARNTZ, R. \& PICHT, H. Introducción a la Terminología. Madrid: Fundación Germán Sánchez Ruipérez, 1995.

CABRÉ, M. T. La terminología: Teoría, metodología, aplicaciones. $1^{\text {a }}$ ed. Barcelona: Empuriés, 1993.

. La terminología: Representación y comunicación. $1^{\mathrm{a}}$ ed. Barcelona: IULA, Universitat Pompeu Fabra, 1999.

CID LEAL, P., y RECODER SELLARÉS, M. J. "Dónde están las llaves o los recursos digitales para la traducción". Anuari de biblioteconomia i informació: Bibliodoc 2000. Barcelona: Collegi Oficial de Bibliotecaris-Documentalistes de Catalunya, 2000. p. 51-68.

GODDARD, R. D., et al. "Collective Efficacy Beliefs: Theoretical Developments, Empirical Evidence, and Future Directions". Educational Researcher, 33(3), 2004. p. 3-13. <www.aera.net/uploadedFiles/Journals_and_Publications/ Journals/Educational_Researcher/Volume_33_No_3/02ERv33n3-Goddard.pdf $>$

HÖNIG, H.G. "Wissen Übersetzer eigentlich, was sie tun?" Lebende Sprachen, 33 (1), 1988. p. 10-14.

HUICI, C. y MORALES, J. F. Psicología de Grupos I. Estructura y Procesos. Madrid: UNED, 2004.

IRAZAZÁBAL NERPELL, A. "Terminología y Documentación”. Jornada Panlatina de Terminología: Perspectives i camps d'aplicació, 1996. Realiter. 3 mayo 2010. <www.realiter.net/spip.php?article764>.

JÄÄSKELÄINEN, R. "The Role of Reference Material in Professional vs. NonProfessional Translation: A Think-Aloud Protocol Study”. Empirical Studies in Translation and Linguistics. Joensuu: University of Joensuu, 1989. p. 175-200. 
KIM, H. "The Influence of Background Information in Translation: Quantity vs. Quality or Both?” Meta, 51(2), 2006. p. 328-342.

KIRALY, D. Pathways to translation. Pedagogy and process. Kent: Kent State University Press, 1995.

KÖNIGS, F.G. Beim Übersetzen schreibt man- übersetzt man auch beim Schreiben? Ein psycholinguistisch orientierter Vergleich zweier fremdsprachlicher Produktionsprozesse bei fortgeschrittenen deutschen Spanischlernern. Ruhr-Universität Bochum: Habilitationsschrift, 1989.

KRINGS, H. P. Was in den Köpfen von Übersetzern vorgeht. Tübingen: Narr, 1986.

KÜNZLI, A. "Experts versus novices: l'utilisation de sources d'information pendant le processus de traduction”. Meta, 2001. V. 46(3), p. 507-523.

KUßMAUL, P. Training the translator. Amsterdam: John Benjamins, 1995.

MAYORAL ASENSIO, R. "La traducción especializada como operación de documentación”. Sendebar, 8-9, 1997-1998. p.137-154.

PICHT, H.. "La enseñanza en la Terminología: un factor decisivo en la formación profesional de personas implicadas en la comunicación y transferencia de conocimiento en una empresa”. R. Gallardo San Salvador y D. Sánchez (eds.), 1992. p. 27-43.

. "La práctica terminológica del traductor". Curso de Invierno: La Terminología: Un mal necesario o una disciplina con perspectivas para estudiantes con inquietudes. Las Palmas G.C.: Universidad de Las Palmas G.C., 27-30 abril 1998. [Inédito]

PINTO, M. "Documentación para la traducción en la sociedad de la información” XV Coloquio Association Internationale de Bibliologie. Salamanca, AIB. (Ponencia), 2000. Página personal de María Pinto. 15 de junio de 2010. <www. mariapinto.es/web/doc/Documentacion_para_la_traduccion.pdf $>$. 
RODRIGUES, C. "A abordagem processual no estudo da traduçao: una meta-análise qualitativa”. Cadernos de Tradução, 2008. 2(10), 23-57. 5 junio 2010. $<$ www.periodicos.ufsc.br/index.php/traducao/article/view/6143/5701 > .

SÁNCHEZ VALVERDE, M. ¿Cómo lo encuentro? Estrategias de traductores para documentarse en Internet. Proyecto de investigación realizado bajo la supervisión del Dr. Ricardo Muñoz Martín en el marco del Máster en Traducción e Interpretación (perfil investigador) de la Universidad de Granada. 2007.

SEVILLA MUÑOZ, M.y SEVILLA MUÑOZ, J. "Todo lo que busque el traductor está en Internet”. Centro Virtual Cervantes. 2005. Instituto Cervantes 25 abril 2010. < cvc.cervantes.es/trujaman/anteriores/septiembre_05/28092005.htm > .

WÜSTER, E.. "Die vier Dimensionen der Terminologiearbeit". Vortrag während des «Kolloquiums über offene terminologische Fragen» in Germersheim, Okt. 1968. Mitteilungsblatt für Dolmetscher und Übersetzer, 15(2), 1-12. 1969. 